\title{
Lipoprotein(a) as a risk factor for calcific aortic valvulopathy in heterozygous familial hypercholesterolemia
}

\section{Vuorio, Alpo}

2019-02

Vuorio , A , Watts , G F \& Kovanen , P T 2019 , ' Lipoprotein(a) as a risk factor for calcific aortic valvulopathy in heterozygous familial hypercholesterolemia ', Atherosclerosis, vol. 281 , pp. 25-30 . https://doi.org/10.1016/j.atherosclerosis.2018.11.040

http://hdl.handle.net/10138/313761

https://doi.org/10.1016/j.atherosclerosis.2018.11.040

publishedVersion

Downloaded from Helda, University of Helsinki institutional repository.

This is an electronic reprint of the original article.

This reprint may differ from the original in pagination and typographic detail.

Please cite the original version. 
Review article

\title{
Lipoprotein(a) as a risk factor for calcific aortic valvulopathy in heterozygous familial hypercholesterolemia
}

\author{
Alpo Vuorio $^{\mathrm{a}, \mathrm{b}, *}$, Gerald F. Watts ${ }^{\mathrm{c}, \mathrm{d}}$, Petri T. Kovanen ${ }^{\mathrm{e}}$ \\ ${ }^{a}$ Mehiläinen Airport Health Centre, 01530, Vantaa, Finland \\ ${ }^{\mathrm{b}}$ University of Helsinki, Department of Forensic Medicine, O0014, Helsinki, Finland \\ ${ }^{\mathrm{c}}$ School of Medicine, Faculty of Medicine and Health Sciences, University of Western Australia, Perth, Australia \\ ${ }^{\mathrm{d}}$ Lipid Disorders Clinic, Department of Cardiology, Royal Perth Hospital, Perth, Australia \\ ${ }^{\mathrm{e}}$ Wihuri Research Institute, Helsinki, Finland
}

\section{H I G H L I G H T S}

- Lp(a) levels above 30-50 mg/dL associate with calcific aortic valve stenosis.

- he-FH patients have marked lifelong elevation of serum LDL-cholesterol level.

- AVC among he-FH patients is more frequent than in the general population.

- Lp(a) life years is a useful metric of cumulative burden of Lp(a).

- Apo(a) antisense oligonucleotides effectively lower Lp(a) concentration.

\section{A R T I C L E I N F O}

\section{Keywords:}

Familial hypercholesterolemia

lipoprotein(a)

Atherosclerosis

Valvulopathy

Aortic calcification

Aortic stenosis

\begin{abstract}
A B S T R A C T
A large number of epidemiological studies in ethnically diverse populations show that lipoprotein(a) [Lp(a)] levels above $30-50 \mathrm{mg} / \mathrm{dL}$ are significantly associated with calcific aortic valve stenosis, although less so in African Americans. Patients with heterozygous familial hypercholesterolemia (he-FH) have a marked lifelong elevation of serum low-density lipoprotein cholesterol (LDL-C) level, and the prevalence of aortic valve calcification (AVC) is at least two-fold higher among adult he-FH patients compared with healthy controls. Additionally, Lp(a) levels above $50 \mathrm{mg} / \mathrm{dL}$ were recently found to be an independent risk factor for AVC among asymptomatic statin-treated he-FH patients. Given that worldwide an estimated 1.4 billion people have an Lp(a) level over $50 \mathrm{mg} / \mathrm{dL}$, and that one out of 250 individuals has he-FH, then globally about 5 million he-FH patients should have an Lp(a) level higher than $50 \mathrm{mg} / \mathrm{dL}$. However, because Lp(a) levels are, on average, significantly higher in he-FH patients than the general population, the actual number of he-FH patients with such high Lp(a) levels must be even higher.

We proposed recently that $\mathrm{Lp}$ (a) life-years is a useful metric of cumulative burden of risk for atherosclerotic cardiovascular disease (ASCVD), and now posit that this metric may be extended to the development of AVC. The $\mathrm{Lp}$ (a) life-years illustrates the age-dependent exposure to a given Lp(a) level (years x mg/dL). Effective novel pharmacotherapies using apo(a) antisense oligonucleotides (ASOs) or small interfering RNA (siRNA)-based therapies targeting the hepatic expression of apo(a) offer unprecedented potential for significant reduction in the cumulative exposure of the aortic valves to $\mathrm{Lp}(\mathrm{a})$, and need to be tested in controlled clinical trials on the progression of AVC.
\end{abstract}

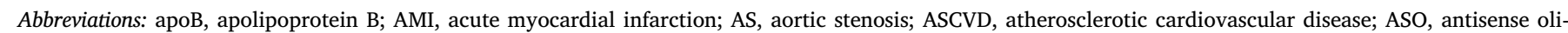

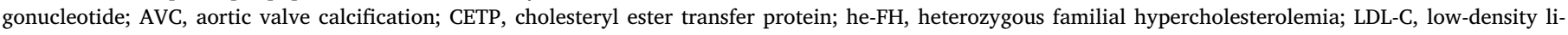

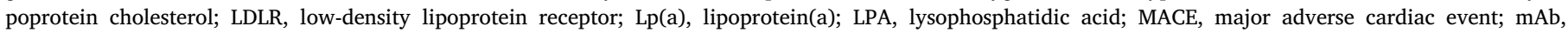

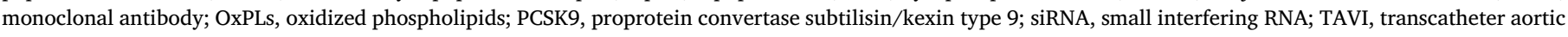
valve implantation

* Corresponding author. Mehiläinen Airport Health Centre, 01530, Vantaa, Finland.

E-mail address: alpo.vuorio@gmail.com (A. Vuorio). 


\section{Introduction}

This review discusses the current knowledge on lipoprotein(a) [Lp (a)] as a risk factor of aortic valve calcification (AVC), an important precursor of clinical aortic stenosis (AS), in heterozygous familial hypercholesterolemia (he-FH) patients, and also takes note of some new treatment options to lower an elevated Lp(a) level. Although the precise etiology of AVC is still unknown [1,2], a recent study found association between genetically determined Lp(a) levels and AVC and incident AS, supporting a causal role for $\mathrm{Lp}(\mathrm{a})$ in the development of the clinical calcific aortic valve disease in the general population [3]. The prevalence of aortic valvular abnormalities increases with age. Indeed, a clear increase in the prevalence of aortic sclerosis, which presents an early stage of the calcific aortic valve disease, is present in about every five persons in the group of 65- to 75-year-old people in the general population [4]. In this age group, the prevalence of clinically significant AS is about $1-3 \%$. Interestingly, in heterozygous familial hypercholesterolemia (he-FH), the figures for AVC, i.e. the stage preceding AS [5], are significantly higher when compared to controls [6], a finding that inspired us to review this topic.

The incidence of a congenital bicuspid valve is approximately $1-2 \%$ [7]. Among the affected individuals, the risk of AS is increased compared with those having a tricuspid, i.e., anatomically a normal valve. Subjects with a bicuspid valve usually develop AS 1 to 2 decades earlier than those with a tricuspid valve, reflecting the importance of blood flow abnormalities in the pathogenesis of AS [8]. For severe AS, the treatment is to replace the aortic valve by open heart surgery, or by transcatheter aortic valve implantation (TAVI), a procedure which has revolutionized the treatment of AS, particularly in elderly patients who are not eligible for major surgery [9].

\section{FH genotype and phenotype}

Familial hypercholesterolemia (FH) is caused by mutations in the low-density lipoprotein receptor $(L D L R)$ gene [10], the apolipoproteinB gene (apoB) [11] or the proprotein convertase subtilisin/kexin type 9 (PCSK9) gene [12]. The vast majority of the mutations causing FH (so far over 1.700) are found in the $L D L R$ gene [13], while the minority of mutations are in the $a p o B$ or PCSK9 gene [14]. The prevalence of heterozygous FH has been traditionally estimated to be one case out of 500 individuals. Recently, however, it has been found that this figure is too conservative, the new estimation for the prevalence of heterozygous $\mathrm{FH}$ (he-FH) being one out of 200-250 persons [15-18].

Patients with he-FH have, on average, a life-long two-fold elevation of serum LDL-cholesterol (LDL-C) level starting already in utero [19]. If left untreated, the elevated LDL-C leads to early onset of atherosclerotic changes in the susceptible areas of the arterial tree, particularly in coronary arteries and the proximal aorta. Indeed, among he-FH patients, angiographically detectable stenosing coronary atherosclerosis has been observed already in 25-year old males and 30-year old females [20], and severe atherothrombotic complications, such as premature acute myocardial infarction (AMI), may occur in male he-FH patients already in early adulthood.

\section{Calcific aortic valvulopathy in the general populations, in African Americans, and in he-FH}

As indicated above, in the general population, the prevalence of AVC increases with age $[21,22]$. In a recent preliminary study involving a small group of patients attending a large general practice for non-cardiac reasons, moderate or severe AS was observed in $5 \%$ of the patients aged $>75$ years [23]. It is important to remember that the severity of AVC is associated with atherosclerotic cardiovascular disease (ASCVD) [24] and that it independently predicts cardiovascular events [25].

Interestingly, race-related differences in AS exist, with African Americans having significantly less likely calcification in the aortic valves than whites. Thus, in a large hospital database study carried out in the US involving 2.1 million patients, African-American individuals had less likely severe aortic stenosis due to degenerative calcific disease (adjusted OR 0.47 [0.36, 0.61]) [26]. In this study, none of the studied known common risk factors of AS could explain the lower prevalence of severe AS among the African Americans. Importantly, however, like in other ethnic groups, the genetic variation of $\mathrm{Lp}(\mathrm{a})$ levelsassociates with AVC and clinical AS also in African Americans [3]. Since in African Americans the levels of Lp(a) are significantly higher than in Caucasians [27], some protective factors counteracting the AVC- and ASpromoting effects of $\mathrm{Lp}(\mathrm{a})$ seem to be operative.

Regarding the manifestations of hypercholesterolemic aortic valvulopathy in $\mathrm{FH}$, both the He-FH and homozygous form of $\mathrm{FH}$ (ho-FH), in which the LDL-C levels are extremely high already in utero, have received early attention [28]. Thus, Buja and co-workers [29] reported that the aortic valve of an autopsied 20-week-old fetus with ho-FH contained numerous lipid-filled cells and fibrous thickening, and that a 9-year-old girl with ho-FH required open-heart surgery for aortic valve replacement. Thus, this pathologic valvular condition in $\mathrm{FH}$ homozygotes appears to represent a greatly accelerated and lipid-dominated, rather than calcification-dominated, form of aortic valvulopathy. Already 20 years ago, Rallidis and co-workers showed, among severely affected he-FH patients a positive correlation between the mean transaortic gradient, severity of aortic valve root involvement, and the [cholesterol x years]-score [30]. However, in those early days, the possible role of $\mathrm{Lp}(\mathrm{a})$ remained unnoticed.

Two studies on the prevalence of AVC in he-FH patients were reported recently (Table 1). Kate et al. [6] employed computed tomography (CT) and estimated AV calcium scores in 145 DNA-verified and/ or clinically diagnosed asymptomatic he-FH patients treated with statins. The prevalence of AVC was $41 \%$ among he-FH patients with a mean age of 52 years and $21 \%$ among controls with a mean age of 56 years (Table 1). This study showed that AVC is about two-fold more frequent among he-FH patients compared with controls. Of particular significance was the comparison between he-FH patients with LDL receptor-negative mutations and those with receptor-defective mutations, the former ones having LDL receptors without any residual functioning capacity to remove LDL particles from the circulation, and the latter ones having LDL receptors with some residual capacity for LDL removal. As expected, LDL-C levels were higher in the former more severely affected group and lower in the latter group of he-FH patients. Strikingly, he-FH patients with LDL receptor-negative mutations had a higher prevalence of AVC (53\%) compared with those with LDL receptor-defective mutations (33\%), and the AVC process advanced faster with age in the LDL receptor-negative patients. In another recent CT scanning study involving 129 DNA-verified and/or clinically diagnosed asymptomatic he-FH patients with a median age of 51 years, $61 \%$ of the patients were found to have AVC [31]. This figure is even higher than that (about 50\%) among subjects over 75 years of age in the general population. Both of the above-cited studies reveal that he-FH patients are likely to have a large extent of subclinical AVC when compared with the subjects in the general population.

Table 1

Aortic valve calcification (AVC) in heterozygous familial hypercholesterolemia (he-FH).

\begin{tabular}{lllll}
\hline Subject of study & Age (years) & Number & $\begin{array}{l}\text { AVC present } \\
\text { Number (\%) }\end{array}$ & Reference \\
\hline Prevalence of AVC & $\begin{array}{llll}\text { he-FH 52 } \pm 8^{\mathrm{a}} \\
\text { controls 56 } \pm 9^{\mathrm{a}}\end{array}$ & 145 & $59(41 \%)$ & {$[6]$} \\
Lp(a) and AVC & $\begin{array}{l}\text { he-FH 51 (46-59) } \\
\text { no controls }\end{array}$ & 129 & $79(61 \%)$ & {$[31]$} \\
& & & & \\
\end{tabular}

AVC $=$ aortic valve calcification

a Mean \pm standard deviation.

b Median (interquartile range). 


\subsection{Inflammatory cells, apolipoproteins, and calcific aortic valvulopathy}

The progress of calcific aortic valvulopathy from early to advanced stenotic valvular lesions has many similarities with atherosclerosis. Thus, it is caused by a multitude of inflammatory reactions and processes particularly on the aortic side of the valve in which turbulent flow conditions exist [1,4,32]. In addition, oxidative stress and endothelial dysfunction are essential parts of the pathogenesis of calcific aortic valvulopathy leading to fibrotic remodelling and calcification of the aortic valve [5]. The early lesions contain infiltrates of inflammatory cells, notably of macrophages, and also of some $\mathrm{T}$ cells [33], and in advanced lesions also mast cells are found [34]. Immunohistochemical studies have shown that already the early lesions, but not the normal regions of the valve leaflets, contain several subclasses apolipoproteins (apo's), notably apo(a) reflecting the presence of $\mathrm{Lp}(\mathrm{a})$, and also apoB and apoE, all of these apolipoproteins indicating the presence of atherogenic and proinflammatory plasma-derived lipoproteins in the early valvular lesions [32]. In the valves with very advanced lesions causing severe AS, also apoA-I is present, reflecting the presence of HDL-particles, which may have, in addition to anti-inflammatory effects, also an anti-calcifying effect in human aortic valves [35]. Taken together, both infiltration of inflammatory cells and accumulation of lipoprotein-derived lipids participate in the initiation and progression of AS.

\subsection{Lipoprotein(a) and calcific aortic valvulopathy in he-FH}

In the general population, increased levels of $\mathrm{Lp}(\mathrm{a})$ in serum are related not only to aortic valve stenosis, but also to aortic valve lesions before a clinically significant AS develops [32,36-41]. It has also been shown that genetic alterations in the LPA locus, which are reflected in the variation of $L p(a)$ levels, are related to aortic valve calcification and aortic stenosis $[3,42]$. The overall data support the hypothesis that during an extended latent period, continuous plasma exposure of the aortic valve to $\mathrm{Lp}(\mathrm{a})$-associated molecules in the circulation induces inflammation and calcification of the valve [41]. Because patients with he-FH have significantly higher Lp(a) levels than non-FH subjects in the general population [43], they might be potentially at higher risk of premature AS. Relevant to the development of AVC is that in the -mentioned study by Kate et al. [6] on 145 he-FH patients, AVC was present in $26 \%, 48 \%$, and $56 \%$ in the age categories of $40-50$ years, 51-58 years, and 59-70 years, respectively. Thus, an elevated concentration of Lp(a) appears to be a critical etiological component in the pathological process leading to AVC. Further, we can hypothesize that lowering of $\operatorname{Lp}(\mathrm{a})$ concentration is one option to decrease the risk of AVC, and, ultimately, also AS. However, proof of this concept requires testing in long-lasting echocardiographic trials with a potent Lp(a)lowering agent [44].

The serum concentration of $\operatorname{Lp}(\mathrm{a})$ in he-FH was found, for the first time, to be an independent predictor for AVC in a study in which 129 (aged 40-69 years) he-FH patients were examined by computed tomography (CT) [31]. Serum Lp(a) levels were higher in he-FH patients with AVC $43.4 \mathrm{mg} / \mathrm{dL}$ [IQR (interquartile range) 10.6-105.0)] when compared with those in the he-FH patients without AVC $24.5 \mathrm{mg} / \mathrm{dL}$ (IQR 5.5-49.1). AVC was associated with serum Lp(a) concentration, age, body mass index, blood pressure, duration of statin use, cholesterol year score, and coronary artery calcification score. Based on the above finding, the authors concluded that it is possible that an elevated serum $\mathrm{Lp}$ (a) concentration may in later life accelerate the development of AS [31]. Considering that aging significantly increases the level of plasma $\mathrm{Lp}(\mathrm{a})$ [45], such age-related elevation of Lp(a) may also act as a mechanism for AS in the elderly.

\section{3. $L p(a)$ and oxidized phospholipids}

It is widely accepted that the $\operatorname{Lp}(\mathrm{a})$-associated risk for ASCVD is largely caused by the oxidized phospholipids (OxPLs) contained in the $\mathrm{Lp}$ (a) particles, particularly when present in the most atherogenic Lp(a) particles that are associated with both high plasma Lp(a) levels and small apo(a) isoforms [46]. In a recent Doppler echocardiography study of 269 patients, aged 18-82 years, who had mild-to-moderate aortic stenosis (AS) at baseline (the Aortic Stenosis Progression Observation: Measuring Effects of Rosuvastatin or ASTRONOMER study), Lp(a) and OxPL-apoB levels were the two factors independently explaining the progression rate of AS [38]. Importantly, the OxPLs located on apoB100 (OxPL-apoB) of the Lp(a) particle mainly reflect the proinflammatory component of the proatherogenic activity of $\mathrm{Lp}(\mathrm{a})$.

The molecular mechanisms explaining how serum $L p(a)$ leads to development of AVC remain to be explored. Like in atherosclerosis, the mechanism might be related to the fact that $\mathrm{Lp}(\mathrm{a})$ has a high affinity for OxPLs and that it may deliver OxPLs to the valvular tissue $[38,47]$. It is possible that autotaxin (ATX), which is present and overexpressed in a mineralized aortic valve, hydrolyzes OxPL into lysophosphatidic acid (LPA) [48], a molecule that triggers fibrosis [49]. Both OxPL and LPA are proinflammatory and can induce calcification [50]. Based on this hypothesis, the potential target molecules to prevent AVC are listed in Table 2. Additionally, fibrinogen accumulation in the aortic valves has been shown to contribute to the pathogenesis of AS, and partially degraded fibrin has an affinity for $\operatorname{Lp}(a)[51,52]$.

Table 2

Main potential therapeutic targets to prevent aortic valve calcification.

\begin{tabular}{ll}
\hline Potential target molecule $^{\mathrm{a}}$ & Proposed pathogenic mechanism of action \\
\hline Lipoprotein(a) & Carries cholesterol, apoB, apo(a), and Ox (PLs) \\
Oxidized phospholipids (OxPLs) & Induce oxidative stress and are proinflammatory \\
Autotaxin & Generates LPA from OxPLs \\
Lysophospatidic acid (LPA) & Induces fibrosis and calcification \\
\hline
\end{tabular}

a Modified from Ref. [41].

\section{Guidelines and lipoprotein(a)}

The finding that $\mathrm{Lp}(\mathrm{a})$ is an independent risk factor for AS is strongly supported by several genetic studies with patients having genetically elevated levels of Lp(a) [37,40,53-56]. However, it has taken a long time to establish guidelines regarding the measurement of $\mathrm{Lp}(\mathrm{a})$ in specific populations like the he-FH [57], however a consensus appears now to have been reached, although the Lp(a) concentration cutoff level above which active treatment is required is still under debate [58]. The European Society of Cardiology/European Atherosclerosis Society Guidelines for the Management of Dyslipidemias recommend measurement of Lp(a) concentration in all patients with he-FH [59]. Regarding the general population, this recommendation considers the ASCVD risk to be significant when serum Lp(a) concentration is > $50 \mathrm{mg} / \mathrm{dL}$, while The Canadian Cardiovascular Society Guidelines recommend the risk threshold to be $30 \mathrm{mg} / \mathrm{dL}$ [60]. It can be argued that, in contrast to the general population, a more stringent treatment goal for $\mathrm{Lp}(\mathrm{a})$ is needed in he-FH, i.e., a risk threshold and treatment goal under $30 \mathrm{mg} / \mathrm{dL}$ appears justified [61]. Actually, since all adult he-FH patients are high-risk patients, the elderly he-FH patients often being even very-high-risk patients, it appears that lowering the serum Lp (a) concentration as much as possible would be appropriate by analogy to the very demanding strategy of lowering LDL-C concentration in these particular patients.

The recent recommendation by the National Heart, Lung, and Blood Institute (NHLBI) working group estimated that the global number of people with an Lp (a) concentration over $50 \mathrm{mg} / \mathrm{dL}$ is about 1.4 billion [44]. If we estimate that about every 250th individual has he-FH, then approximately 5 million he-FH patients having an Lp(a) level over $50 \mathrm{mg} / \mathrm{dL}$ should exist worldwide. Because patients with he-FH have significantly higher Lp(a) levels than the non-FH subjects in the general 
population [43], the number of he-FH patients with such high Lp(a) levels must be even greater.

\section{Lipoprotein(a) life-years as an exemplar of CV risk in he-FH}

We recently proposed the possibility of using "Lp(a) life-years" as another metric of cumulative burden of ASCVD risk in patients with heFH [61]. Since Lp(a) appears to be a pathogenic component of AVC, this metric can be extended to include this disease, as well. Lp(a) life-years illustrates the age-dependent exposure to $\mathrm{Lp}(\mathrm{a})$ of a given concentration (years $\mathrm{x} \mathrm{mg/dL}$ ). The analogy for using Lp(a) life-years in he-FH is based on the use of LDL-C life-years to illustrate the lifelong burden of a more or less stable genetically increased level of LDL-C (years $\mathrm{x} \mathrm{mg/dL}$ or $\mathrm{mmol} / \mathrm{L}$ ) in he-FH [62]. Like the plasma levels of increased LDL-C in he-FH, those of Lp(a) are not constant, as they increase with age, and may also fluctuate, especially in patients with liver and kidney disease [63]. Thus, a single Lp(a) value may be misleading when extrapolated to calculate Lp(a) life-years. Accordingly, the plasma level of Lp(a) should be measured several times, and the mean value then used to make a more reliable estimation of an actual life-long situation. If an elevated $\mathrm{Lp}(\mathrm{a})$ is pharmacologically lowered, as it is currently possible, it is advisable to measure the $\mathrm{Lp}(\mathrm{a})$ level on an annual basis.

Fig. 1A illustrates cumulative Lp(a) life-years up to the age of 80 years of a person having an $\mathrm{Lp}(\mathrm{a})$ level of either $30 \mathrm{mg} / \mathrm{dL}$ or $50 \mathrm{mg} / \mathrm{dL}$, i.e. levels which correspond to the cut-off levels based on the recommendation of the Canadian Cardiovascular Society Guidelines [60] and The European Society of Cardiology/European Atherosclerosis Society [59], respectively. The figure depicts that a person having an Lp (a) level of $30 \mathrm{mg} / \mathrm{dL}$ will reach an Lp(a) life-years value of 2400 (years $\mathrm{x} \mathrm{mg/dL),} \mathrm{while} \mathrm{a} \mathrm{person} \mathrm{having} \mathrm{an} \mathrm{Lp(a)} \mathrm{level} \mathrm{of} 50 \mathrm{mg} / \mathrm{dL}$ will reach an $\mathrm{Lp}$ (a) life-years value which is much higher, i.e., 4000 (years $\mathrm{x} \mathrm{mg}$ / $\mathrm{dL})$, when he/she reaches the age of 80 years.

Fig. 1B shows the cumulative Lp(a) life-years when an individual has a very high $(100 \mathrm{mg} / \mathrm{dL}) \mathrm{Lp}(\mathrm{a})$ serum concentration. Based on the Canadian recommendation, this individual reaches the recommended maximum Lp(a) life-years already at the age of about 25 years. According to a recent CT study among he-FH patients, an $\mathrm{Lp}$ (a) level of $30 \mathrm{mg} / \mathrm{dL}$ or less was not a risk for AVC, but Lp(a) levels over $50 \mathrm{mg} / \mathrm{dL}$ posed a significant risk for AVC [31]. Regarding the above-mentioned high-risk individual, an early Lp(a) lowering therapy would be very appropriate, since if such treatment is started in late adulthood, the cumulative $\mathrm{Lp}$ (a) burden has already reached an excessive level. In summary, by analogy with rescue therapy of an untreated middle-aged
he-FH patient with high cumulative burden of LDL-C [64], an adult heFH patient with a high Lp(a) serum concentration (in addition to the high LDL-C level) needs very effective rescue therapy to lower the cumulative burden of $\mathrm{Lp}(\mathrm{a})$.

\section{Challenges related to Lp(a) lowering therapies}

In a very recent meta-analysis including 29,069 patients, who had participated in randomised placebo-controlled statin trials, those with an elevated Lp(a) level of $30 \mathrm{mg} / \mathrm{dL}$ or higher at baseline and those with elevated $\mathrm{Lp}$ (a) level of $50 \mathrm{mg} / \mathrm{dL}$ or higher while on statin treatment, showed an independent linear relation with risk of CV events [65]. These data show that a residual risk remains in patients with an elevated $\mathrm{Lp}$ (a) (mainly $>50 \mathrm{mg} / \mathrm{dL}$ ) even while taking statins, and clearly calls for additional trials to test for specific therapies to lower Lp (a).

Several issues need to be taken into account before implementing effective rescue therapies in the routine clinical care of he-FH patients. The most important clinical challenges are listed in Table 3 . The very recent data of the Odyssey Outcomes Trial regarding the effects of the PCSK9 inhibitor alirocumab on serum Lp(a) concentration and on cardiovascular outcomes are interesting [66]. In this outcome trial on patients with recent major adverse cardiac event (MACE), it was found that $\mathrm{Lp}$ (a) lowering by alirocumad is associated with a reduction of MACEs, and that reduction is independent of baseline LDL-C level and concurrent LDL-C lowering. The considerable Lp(a)-lowering effect of PCSK9-mAbs seems not to be related to the mAb type, either alirocumab or evolocumab, as in a recent meta-analysis including twentyseven randomised clinical trials with 11,864 participants PCSK9-mAbs were found to significantly reduce $\operatorname{Lp}$ (a) $(-21.9 \%$; $95 \%$ CI -24.3 to -19.5) [67]. It remains to be seen whether the PCSK9-mAbs will reduce not only MACEs but also the development of AS.

\section{Conclusions}

All he-FH patients have a lifelong two- to three-fold elevation of LDL-C levels, and a large proportion of them also have a lifelong elevation of Lp(a) level. The combination of an elevated LDL-C and an elevated Lp(a) level in he-FH patients not only increases the risk of ASCVD, but also potentially also the risk of calcific aortic valvulopathy. The concept of Lp(a) life-years emphasizes the lifelong burden of elevated serum $\mathrm{Lp}(\mathrm{a})$ level and the need to lower serum $\mathrm{Lp}$ (a) concentration effectively in he-FH, since in such patients the circulating

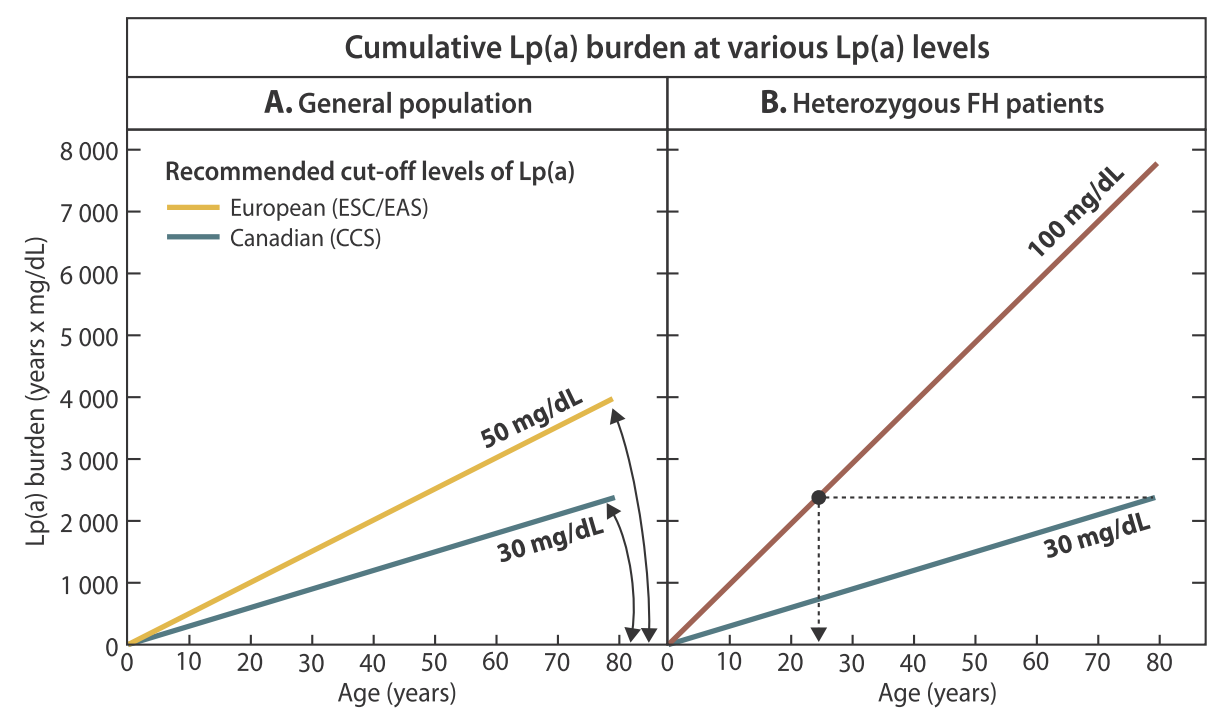

Fig. 1. Cumulative Lp(a) burden.

(A) Cumulative $\mathrm{Lp}(\mathrm{a})$ burden (years $\mathrm{x} \mathrm{mg} / \mathrm{dL}$ ) up to the age of 80 years of a person having an Lp(a) level of either $30 \mathrm{mg} / \mathrm{dL}$ or $50 \mathrm{mg} / \mathrm{dL}$, i.e., the cut-off level above which active treatment is required is based on the recommendation of the Canadian Cardiovascular Society (CCS) Guidelines [60] and The European Society of Cardiology (ESC)/European Atherosclerosis Society (EAS) [59], respectively. (B) Cumulative Lp(a) burden in two he-FH patients, one having a very high $(100 \mathrm{mg} / \mathrm{dL})$ and another having a relatively low $(30 \mathrm{mg} / \mathrm{dL}) \mathrm{Lp}(\mathrm{a})$ serum concentration. The $\mathrm{Lp}(\mathrm{a})$ concentrations are based on the mean of two separate measurements. The he-FH patient with the high Lp(a) concentration reaches the cumulative Lp(a) burden of the 80-year old heFH patient with the relatively low $L p(a)$ level already at the age of about 25 years. According to a recent computed tomography study in he-FH patients, Lp(a) levels over $50 \mathrm{mg} / \mathrm{dL}$ pose a significant risk for AVC, but levels of $30 \mathrm{mg} / \mathrm{dL}$ or less do not [31]. Regarding the above presented high-risk he-FH patient, Lp(a) lowering therapy would be very appropriate. 
Table 3

Key challenges related to Lp(a) lowering therapy in he-FH.

Clinical challenge
Lack of physician awareness of value of Lp(a)
Perception that Lp(a) assay is not standardised and is heavily dependent on apo(a)
isoform size
No internationally accepted target goal for the treatment
Need for potent therapies enabling effective lowering of Lp(a)
Lack of clinical trials showing effect of Lp(a) lowering therapy on outcomes in he-FH
Belief that Lp(a) is no more a risk factor for ASCVD once LDL-C is decreased to very
low levels ${ }^{\mathrm{a}}$

a $[66]$.

levels of two artery-damaging lipoprotein particles, i.e. LDL particles and Lp(a) particles, are elevated since birth. Especially, the new apo(a) antisense oligonucleotides which lower serum Lp(a) concentration by about $70-90 \%$ are very promising to meet this challenge. These new apo(a) antisense oligonucleotides, and also the specific siRNAs under development, all need to be tested in controlled clinical intervention trials to learn about their effects on the progression of ASCVD [68-73]. In he-FH patients, this promising mode of selective Lp(a)-lowering therapy has the prospects of having a double target: both the atherosclerosis-susceptible areas of the arterial wall and the aortic valves. Such therapy would be probably most efficient among those he-FH patients who have a strongly elevated Lp(a) level and early signs of calcific aortic valvulopathy.

\section{Conflict of interest}

The authors declared they do not have anything to disclose regarding conflict of interest with respect to this manuscript.

\section{References}

[1] F.G. Bakaeen, T.K. Rosengart, B.A. Carabello, Aortic stenosis, Ann. Intern. Med. 166 (2017) ITC1.

[2] Brian R. Lindman, Aortic Stenosis. JACC (Journal of the American College of Cardiology) 69 (2017) 1533-1535.

[3] G. Thanassoulis, C.Y. Campbell, D.S. Owens, G. Smith, A.V. Smith, G.M. Pelos, et al., Genetic associations with valvular calcification and aortic stenosis, N. Engl. J. Med. 368 (2013) 503-512.

[4] B.A. Carabello, W.J. Paulus, Aortic stenosis, Lancet 373 (2009) 956-966.

[5] M.M. Izquierdo-Gómez, I. Hernández-Betancor, J. García-Niebla, B. Marí-López, L. Laynez-Cerdeña, J. Lacalzada-Almeida, Valve calcification in aortic stenosis: etiology and diagnostic imaging techniques, BioMed Res. Int. 2017 (2017) 1-12.

[6] ten Kate, R. Gert-Jan, S. Bos, A. Dedic, Neefjes, A. Lisan, et al., Increased aortic valve calcification in familial hypercholesterolemia, JACC (J. Am. Coll. Cardiol.) 66 (2015) 2687-2695.

[7] S. Chandra, N.M. Rajamannan, P. Sucosky, Computational assessment of bicuspid aortic valve wall-shear stress: implications for calcific aortic valve disease, Biomechanics Model. Mechanobiol. 11 (2012) 1085-1096.

[8] S.J. Cowell, D.E. Newby, N.A. Boon, A.T. Elder, Calcific aortic stenosis: same old story? Age Ageing 33 (2004) 538-544.

[9] B.R. Lindman, R.O. Bonow, C.M. Otto, Current management of calcific aortic stenosis, Circ. Res. 113 (2013) 223-237.

[10] J.L. Goldstein, M.S. Brown, The LDL receptor locus and the genetics of familial hypercholesterolemia, Annu. Rev. Genet. 13 (1979) 259-289.

[11] T.L. Innerarity, K.H. Weisgraber, K.S. Arnold, R.W. Mahley, R.M. Krauss, G.L. Vega, et al., Familial defective apolipoprotein B-100: low density lipoproteins with abnormal receptor binding, Proc. Natl. Acad. Sci. U.S.A. 84 (1987) 6919-6923.

[12] P. Moulin, L. Wickham, E. Bruckert, J. Lecerf, M. Krempf, M. Varret, et al, Mutations in PCSK9 cause autosomal dominant hypercholesterolemia, Nat. Genet. 34 (2003) 154-156.

[13] S. Leigh, M. Futema, R. Whittall, A. Taylor-Beadling, M. Williams, J.T. den Dunnen, et al., The UCL low-density lipoprotein receptor gene variant database: pathogenicity update, J. Med. Genet. 54 (2017) 217-223 2016.

[14] S.E. Humphries, T. Cranston, M. Allen, H. Middleton-Price, M.C. Fernandez, V. Senior, et al., Mutational analysis in UK patients with a clinical diagnosis of familial hypercholesterolaemia: relationship with plasma lipid traits, heart disease risk and utility in relative tracing, J. Mol. Med. 84 (2006) 203-214.

[15] M. Benn, G.F. Watts, A. Tybjærg-Hansen, B.G. Nordestgaard, Mutations causative of familial hypercholesterolaemia: screening of 98098 individuals from the Copenhagen General Population Study estimated a prevalence of 1 in 217, Eur. Heart J. 37 (2016) 1384-1394.

[16] A.V. Khera, H. Won, G.M. Peloso, K.S. Lawson, T.M. Bartz, X. Deng, et al.,
Diagnostic yield and clinical utility of sequencing familial hypercholesterolemia genes in patients with severe hypercholesterolemia, J. Am. Coll. Cardiol. 67 (2016) $2578-2589$.

[17] J. Pang, P.J. Lansberg, G.F. Watts, Department of Vascular Medicine, University of Western Australia, Royal Perth Hospital, et al. International developments in the care of familial hypercholesterolemia: where now and where to next? J. Atherosclerosis Thromb. 23 (2016) 505-519.

[18] D.S. Wald, J.P. Bestwick, J.K. Morris, K. Whyte, L. Jenkins, N.J. Wald, Child-parent familial hypercholesterolemia screening in primary care, N. Engl. J. Med. 375 (2016) 1628-1637.

[19] A.F. Vuorio, H. Turtola, K. Kontula, Neonatal diagnosis of familial hypercholesterolemia in newborns born to a parent with a molecularly defined heterozygous familial hypercholesterolemia, Arterioscler. Thromb. Vasc. Biol. 17 (1997) 3332-3337.

[20] H. Mabuchi, J. Koizumi, M. Shimizu, R. Takeda, Development of coronary heart disease in familial hypercholesterolemia, Circulation 79 (1989) 225-232.

[21] M. Lindroos, M. Kupari, J. Heikkilä, R. Tilvis, Prevalence of aortic valve abnormalities in the elderly: an echocardiographic study of a random population sample, $\mathrm{J}$. Am. Coll. Cardiol. 21 (1993) 1220-1225.

[22] K. Nasir, R. Katz, J. Takasu, D.M. Shavelle, R. Detrano, J.A. Lima, et al, Ethnic differences between extra-coronary measures on cardiac computed tomography: multi-ethnic study of atherosclerosis (MESA), Atherosclerosis 198 (2008) 104-114.

[23] C. Williams, N. Maskell, E. Rees, K. Truman, A. Ionescu, Prevalence of aortic valve abnormalities in the elderly: a screening study using hand-held ultrasound scanning, Heart 102 (Suppl 6) (2016) A113 (Abstract 157).

[24] A. Rossi, P. Faggiano, A.E. Amado, M. Cicoira, S. Bonapace, L. Franceschini, et al., Mitral and aortic valve sclerosis/calcification and carotid atherosclerosis: results from 1065 patients, Heart Ves. 29 (2014) 776-783.

[25] D.S. Owens, M.J. Budoff, R. Katz, Junichiro Takasu, D.M. Shavelle, J Jeffrey Carr, et al., Aortic valve calcium independently predicts coronary and cardiovascular events in a primary prevention population, JACC (J. Am. Coll. Cardiol.): Cardiovascular Imaging 5 (2012) 619-625.

[26] D.K. Patel, K.D. Green, M. Fudim, F.E. Harrell, T.J. Wang, M.A. Robbins, Racial differences in the prevalence of severe aortic stenosis, Journal of American Heart Association 3 (2014) e000879.

[27] S.M. Marcovina, J.J. Albers, D.R. Jacobs Jr., L.L. Perkins, C.E. Lewis, B.V. Howard, et al., Lipoprotein[a] concentrations and apolipoprotein[a] phenotypes in Caucasians and African Americans. The CARDIA study, Arterioscler. Thromb. 13 (1993) 1037-1045.

[28] A. Kawaguchi, C. Yutani, A. Yamamoto, Hypercholesterolemic valvulopathy: an aspect of malignant atherosclerosis, Ther. Apher. Dial. 7 (2003) 439-443.

[29] L.M. Buja, P.T. Kovanen, D.W. Bilheimer, Cellular pathology of homozygous familial hypercholesterolemia, Am. J. Pathol. 97 (1979) 327-357.

[30] L. Rallidis, R.P. Naoumova, G.R. Thompson, P. Nihoyannopoulos, Extent and severity of atherosclerotic involvement of the aortic valve and root in familial hypercholesterolaemia, Heart 80 (1998) 583-590.

[31] R. Vongpromek, S. Bos, G.-R. Kate, R. Yahya, A.J.M. Verhoeven, P.J. Feyter, et al., Lipoprotein(a) levels are associated with aortic valve calcification in asymptomatic patients with familial hypercholesterolaemia, J. Intern. Med. 278 (2015) 166-173.

[32] K.D. O'Brien, D.D. Reichenbach, S.M. Marcovina, J. Kuusisto, C.E. Alpers, C.M. Otto B. Apolipoproteins, (a), and E accumulate in the morphologically early lesion of 'degenerative' valvular aortic stenosis, Arterioscler. Thromb. Vasc. Biol. 16 (1996) $523-532$.

[33] C.M. Otto, B. Prendergast, Aortic-valve stenosis-from patients at risk to severe valve obstruction, N. Engl. J. Med. 21 (371) (2014) 744-756.

[34] S. Helske, S. Syväranta, M. Kupari, J. Lappalainen, M. Laine, J. Lommi, et al., Possible role for mast cell-derived cathepsin $\mathrm{G}$ in the adverse remodelling of stenotic aorticvalves, Eur. Heart J. 27 (2006) 1495-1504.

[35] J.I. Lommi, P.T. Kovanen, M. Jauhiainen, M. Lee-Rueckert, M. Kupari, S. Helske, High-density lipoproteins (HDL) are present in stenotic aortic valves and may interfere with the mechanisms of valvular calcification, Atherosclerosis 219 (2011) $538-544$.

[36] B.F. Stewart, D. Siscovick, B.K. Lind, J.M. Gardin, J.S. Gottdiener, V.E. Smith, et al., Clinical factors associated with calcific aortic valve disease. Cardiovascular Health Study, JACC (J. Am. Coll. Cardiol.) 29 (1997) 630-634.

[37] P.R. Kamstrup, A. Tybjærg-Hansen, B.G. Nordestgaard, Elevated lipoprotein(a) and risk of aortic valve stenosis in the general population, JACC (J. Am. Coll. Cardiol.) 63 (2014) 470-477.

[38] R. Capoulade, K.L. Chan, C. Yeang, P. Mathieu, Y. Bossé, J.G. Dumesnil, et al, Oxidized phospholipids, lipoprotein(a), and progression of calcific aortic valve stenosis, JACC (J. Am. Coll. Cardiol.) 66 (2015) 1236-1246.

[39] J. Cao, B.T. Steffen, M. Budoff, W.S. Post, G. Thanassoulis, B. Kestenbaum, et al., Lipoprotein(a) levels are associated with subclinical calcific aortic valve disease in Caucasian and Black individuals: the Multi-Ethnic Study of Atherosclerosis, Arterioscler. Thromb. Vasc. Biol. 36 (2016) 1003-1009.

[40] G. Thanassoulis, Lipoprotein (a) in calcific aortic valve disease: from genomics to novel drug target for aortic stenosis, JLR (J. Lipid Res.) 57 (2016) 917-924.

[41] M. Torzewski, A. Ravandi, C. Yeang, A. Edel, R. Bhindi, S. Kath, et al., Lipoprotein (a) associated molecules are prominent components in plasma and valve leaflets in calcific aortic valve stenosis, JACC Basic to Translational Science 2 (2017) 229-240.

[42] S. Burgess, B.A. Ference, J.R. Staley, D.F. Freitag, A.M. Mason, S.F. Nielsen, et al., Association of LPA variants with risk of coronary disease and the implications for lipoprotein(a)-lowering therapies: a mendelian randomization analysis, JAMA Cardiology (2018), https://doi.org/10.1001/jamacardio.2018.1470 ([Epub ahead of print]).

[43] Utermann G, F. Hoppichler, H. Dieplinger, M. Seed, G. Thompson, E. Boerwinkle, 
Defects in the low density lipoprotein receptor gene affect lipoprotein (a) levels: multiplicative interaction of two gene loci associated with premature atherosclerosis, Proc. Natl. Acad. Sci. U. S. A. 86 (1989) 4171-4174.

[44] S. Tsimikas, S. Fazio, K.C. Ferdinand, H.N. Ginsberg, M.L. Koschinsky, S.M. Marcovina, et al., NHLBI Working Group recommendations to reduce lipoprotein(a)-mediated risk of cardiovascular disease and aortic stenosis, J. Am. Coll. Cardiol. 71 (2018) 177-192.

[45] H. Akita, M. Matsubara, H. Shibuya, H. Fuda, H. Chiba, Effect of ageing on plasma lipoprotein(a) levels, Ann. Clin. Biochem. 39 (2002) 237-240.

[46] A. Taleb, J.L. Witztum, S. Tsimikas, Oxidized phospholipids on apoB-100-containing lipoproteins: a biomarker predicting cardiovascular disease and cardiovascular events, Biomark. Med. 5 (2011) 673-694.

[47] R. Bouchareb, A. Mahmut, M.J. Nsaibia, M.C. Boulanger, A. Dahou, J.L. Lepine, et al., Autotaxin derived from lipoprotein(a) and valve interstitial cells promotes inflammation and mineralization of the aortic valve, Circulation 132 (2015) 677-690.

[48] M.J. Nsaibia, A. Mahmut, M. Boulanger, B.J. Arsenault, R. Bouchareb, S. Simard, et al., Autotaxin interacts with lipoprotein(a) and oxidized phospholipids in predicting the risk of calcific aortic valve stenosis in patients with coronary artery disease, J. Intern. Med. 280 (2016) 509-517.

[49] P. Mathieu, M.C. Boulanger, R. Bouchareb, Molecular biology of calcific aortic valve disease: towards new pharmacological therapies, Expert Rev. Cardiovasc. Ther. 12 (2014) 851-862.

[50] C. Yeang, M.J. Wilkinson, S. Tsimikas, Lipoprotein(a) and oxidized phospholipids in calcific aortic valve stenosis, Curr. Opin. Cardiol. 31 (2016) 440-450.

[51] K.W. Walton, N. Williamson, A.G. Johnson, The pathogenesis of atherosclerosis of the mitral and aortic valves, J. Pathol. 101 (1970) 205-220.

[52] R.M. Lawn, Lipoprotein(a) in heart disease, Sci. Am. 266 (1992) 54-60.

[53] B.J. Arsenaut, S.M. Boekholdt, M.P. Dubé, et al., Lipoprotein(a) levels, genotype and incident aortic valve stenosis: a prospective mendelian randomization study and replication in a case-control cohort, Circulation Cardiovascular Genetics 7 (2014) 304-310.

[54] M. Hung, J.L. Witztum, S. Tsimikas, New therapeutic targets for calcific aortic valve stenosis: the lipoprotein(a)-lipoprotein-associated phospholipase A2-oxidized phospholipid Axis, JACC (J. Am. Coll. Cardiol.) 63 (2014) 478-480.

[55] B.J. Cairns, S. Coffey, R.C. Travis, B. Prendergast, J. Green, J.C. Engert, et al., A replicated, genome-wide significant association of aortic stenosis with a genetic variant for lipoprotein(a): meta-analysis of published and novel data, Circulation 135 (2017) 1181-1183.

[56] H.Y. Chen, L. Dufresne, H. Burr, A. Ambikkumar, N. Yasui, K. Luk, et al., Association of LPA variants with aortic stenosis: a large-scale study using diagnostic and procedural codes from electronic health records, JAMA Cardiol 3 (2018) 18-23.

[57] K.M. Kostner, W. März, G.M. Kostner, When should we measure lipoprotein (a)? Eur. Heart J. 34 (2013) 3268-3276.

[58] G.M. Kostner, K.M. Kostner, A.S. Wierzbicki, Is Lp(a) ready for prime time use in the clinic? A pros-and-cons debate, Atherosclerosis 274 (2018) 16-22.

[59] A.L. Catapano, I. Graham, G. De Backer, O. Wiklund, M.J. Chapman, H. xel, et al., 2016 ESC/EAS Guidelines for the management of dyslipidaemias, Eur. Heart J. 37
(2016) 2999-3058.

[60] T.J. Anderson, J. Grégoire, G.J. Pearson, A.R. Barry, P. Couture, M. Dawes, et al., Canadian Cardiovascular Society Guidelines for the management of dyslipidemia for the prevention of cardiovascular disease in the adult, Can. J. Cardiol. 32 (2016) 1263-1282.

[61] A. Vuorio, G.F. Watts, P.T. Kovanen, Depicting new pharmacological strategies for familial hypercholesterolaemia involving lipoprotein (a), Eur. Heart J. 38 (2017) 3555-3559.

[62] A. Vuorio, K.F. Docherty, S.E. Humphries, J. Kuoppala, P.T. Kovanen, Statin treatment of children with familial hypercholesterolemia - trying to balance incomplete evidence of long-term safety and clinical accountability: are we approaching a consensus? Atherosclerosis 226 (2013) 315-320.

[63] L.M. de Boer, M.H. Hof, A. Wiegman, J.J. Kastelein, B.A. Hutten, Are lipoprotein(a) levels constant over time? A follow-up study of a large cohort of children referred to a pediatric lipid clinic, Atherosclerosis Suppl. 32 (2018).

[64] A. Vuorio, G.F. Watts, P.T. Kovanen, Rescue therapy with PCSK9 inhibitors for patients with delayed diagnosis of heterozygous familial hypercholesterolemia: redressing the balance of missed opportunities, Journal of Clinical Lipidology 10 (2016) 1278-1279.

[65] P. Willeit, P.M. Ridker, P.J. Nestel, J. Simes, A.M. Tonkin, T.R. Pedersen, et al. Baseline and on-statin treatment lipoprotein(a) levels for prediction of cardiovascular events: individual patient-data meta-analysis of statin outcome trials, Lancet 392 (2018) 1311-1320.

[66] V. Bittner, M. Szarek, P.E. Aylward, D.L. Bhatt, R. Diaz, Z. Fras, et al., Lp(a) and cardiovascular outcomes: an analysis from the Odyssey outcomes trial, Atherosclerosis Suppl. 32 (2018) 24-25.

[67] Y. Cao, H. Liu, S. Li, J. Li, A Meta-Analysis of the Effect of PCSK9-Monoclonal antibodies on circulating lipoprotein (a) levels, Am. J. Cardiovasc. Drugs (2018 Sep 19), https://doi.org/10.1007/s40256-018-0303-2 [Epub ahead of print].

[68] S. Tsimikas, N.J. Viney, S.G. Hughes, W. Singleton, M.J. Graham, B.F. Baker, et al., Antisense therapy targeting apolipoprotein(a): a randomised, double-blind, placebo-controlled phase 1 study, Lancet 386 (2015) 1472-1483.

[69] Sotirios Tsimikas, The re-emergence of lipoprotein(a) in a broader clinical arena, Prog. Cardiovasc. Dis. 59 (2016) 135-144.

[70] S. Tsimikas, A Test in Context: lipoprotein(a): diagnosis, prognosis, controversies, and emerging therapies, JACC (J. Am. Coll. Cardiol.) 69 (2017) 692-711.

[71] M.J. Graham, N. Viney, R.M. Crooke, S. Tsimikas, Antisense inhibition of apolipoprotein (a) to lower plasma lipoprotein (a) levels in humans, JLR (J. Lipid Res.) 57 (2016) 340-351.

[72] N.J. Viney, van Capelleveen, C. Julian, R.S. Geary, S. Xia, J.A. Tami, R.Z. Yu, et al. Antisense oligonucleotides targeting apolipoprotein(a) in people with raised lipoprotein(a): two randomised, double-blind, placebo-controlled, dose-ranging trials, Lancet 388 (2016) 2239-2253.

[73] Amgen, 2016. Amgen And Arrowhead Pharmaceuticals Announce Two Cardiovascular Collaborations. http://www.amgen.com/media/news-releases/ 2016/09/amgen-and-arrowhead-pharmaceuticals-announce-two-cardiovascularcollaborations/. 